\title{
DIÁLOGO INTRAMUSICAL: INTERACCIÓN, COMUNICACIÓN Y METÁFORA DE LA CONVERSACIÓN EN LA MÚSICA BLUES
}

\author{
INTRAMUSICAL DIALOGUE: INTERACTION, COMMUNICATION \\ AND THE METAPHOR OF CONVERSATION IN BLUES MUSIC
}

\author{
Josep PEDRO \\ Universidad Complutense de Madrid \\ jpedro@ucm.es
}

Resumen: Partiendo de una investigación etnográfica y analítica sobre las escenas de blues en Austin, Texas y en Madrid, este artículo plantea el concepto de diálogo intramusical con el objetivo de indagar en las formas de comunicación dialógica que se establecen en la interpretación musical. Reflexionamos sobre la idea del blues como lenguaje musical regido por un sistema de convenciones y abordamos la metáfora de la interpretación como conversación a partir de los discursos de diversos músicos entrevistados.

Palabras clave: Diálogo intramusical. Interacción. Comunicación. Metáfora de la conversación. Blues.

Abstract: Based on an ethnographic and analytical research about the
blues scenes in Austin, Texas and Madrid, this paper posits the concept
of intramusical dialogue in order to explore the forms of dialogic
communication that are established in musical performance. We will
discuss the idea of blues as a musical language regulated by a system 
of conventions, and we will approach the metaphor of performance as conversation by drawing on the discourses of different musicians that have been interviewed throughout the research.

Key Words: Intramusical dialogue. Interaction. Communication. Metaphor of conversation. Blues.

\section{INTRODUCCIÓN}

Este artículo parte del interés por las formas de comunicación dialógica que se establecen en la interpretación musical entre los intérpretes de un determinado grupo; los intérpretes y los públicos; y entre los intérpretes, los públicos y las tradiciones complejas y unificadoras de géneros musicales como el blues. Para entender y analizar estos procesos recurrimos al concepto de "diálogo intramusical", nos apoyamos en la investigación de tesis (en curso) realizada sobre las escenas de blues en Austin (Texas) y Madrid, y asumimos que la música es un lenguaje que utilizamos para expresar sentimientos, emociones e ideas; para comunicarnos, situarnos en el mundo y transformarlo ${ }^{1}$. Así, el aprendizaje y la interpretación del blues suponen familiarizarse con un tipo de lenguaje musical particular - un sistema de convenciones que implica determinadas reglas, lógicas y posibilidades.

La idea de "lenguaje musical" ha sido ampliamente asumida y discutida en el ámbito de la etnomusicología (Cruces, 2001; Keil, 1991; Richardson, 1999) y la educación musical (Peñalver Vilar, 2011, 2013), así como en los discursos sobre música popular (especialmente sobre jazz y blues) reproducidos por investigadores, periodistas, músicos y aficionados. Además, en el ámbito de la comunicación, Antonio Méndez (2016: 54) ha señalado que "la música puede y debe pensarse como otra forma de lenguaje que, a su vez, se cruza en la práctica con otros lenguajes socialmente vivos"2. No obstante, la discusión de la música como lenguaje

1. A lo largo del artículo introduciré los discursos de músicos destacados de las escenas estudiadas, que han sido extraídos de las entrevistas realizadas durante la etnografía presencial en Austin y Madrid. En total he realizado 46 entrevistas en profundidad, 36 presenciales y 10 online. En el artículo me referiré a 7 de ellas (6 presenciales y 1 online; 5 publicadas y 2 inéditas), así como a testimonios recogidos en la observación participante offline y online.

2. Méndez (2016: 72) señala que el lenguaje verbal y la comunicación musical son espacios de 
es todavía variable e imprecisa. En este trabajo empleamos la noción de "lenguaje musical" para referirnos tanto al sistema de convenciones que regula y posibilita la interpretación, el diálogo y la comunicación intramusical, como a la capacidad de expresarse a través de ese sistema de un modo comparable pero diferente al del lenguaje verbal -"hablando", articulando frases, respondiendo y desarrollando, idealmente, una voz personal o propia. $\mathrm{O}$, en otros términos, para referirnos tanto al conjunto de elementos formales y convenciones que definen un género musical como el blues, como a la apropiación y recreación práctica de ese sistema en la interpretación.

La discusión que planteamos desde una perspectiva etnográfica y semiótica se inscribe en la tradición de estudios sobre improvisación, dinámicas participativas y procesos de interacción en la interpretación musical. En este sentido, cabe mencionar la inspiración tomada de investigaciones sobre la improvisación en el jazz (Berliner, 1994; Monson, 2009), debates sobre calidad musical en función de la interacción(Tsioulakis, 2013) y discusiones sobre dinámicas presentacionales y participativas en la performance (Turino, 2008). Asimismo, hay que destacar el trabajo de los sociólogos Robert Faulkner y Howard Becker (2009) sobre el repertorio del jazz. Su pregunta de partida - ¿cómo consigue un grupo de gente, que no ha preparado una actuación musical, darla de manera efectiva? (Faulkner y Becker, 2009: 1)- remite directamente al objetivo planteado, si bien la distinta naturaleza de los músicos estudiados y el interés de estos autores en resaltar el conocimiento mínimo necesario para sacar adelante una actuación musical (en tanto trabajo) plantea diferencias significativas. En cualquier caso, cabe señalar que, tanto en el blues como en el jazz, la interpretación grupal sin ensayos previos no se basa únicamente en el conocimiento común de ciertas canciones de la tradición sino, sobre todo, en la apropiación y negociación práctica del sistema de convenciones que posibilita el acuerdo y guía la interpretación.

\footnotetext{
"aprendizaje compartido, de carácter social, comunicativo, común” y que "la condición musical, como la condición lingüística, es una condición dialógica”, caracterizada por la interacción recíproca y continua entre "música, lenguaje y cultura." No obstante, indica que "música y lenguaje no pueden identificarse sin más aunque sea sólo porque la primera carece de la canalización referencial y semántica que define al segundo" (2016: 56). Como muestra su amplia y cuidadosa discusión (2016: 53-85), la música es un tipo de lenguaje muy particular y complejo, del que han hablado autores ilustres como Darwin, Rousseau o Lévi-Strauss, entre otros. Sin duda, la reflexión sobre música y lenguaje puede conducir a largas discusiones técnicas. No obstante, nuestro principal interés es comprender el modo en que los músicos de las escenas de blues en Austin y Madrid asumen y utilizan la idea de lenguaje musical.
} 
El blues resulta especialmente interesante para investigar la experiencia de la interacción y la comunicación intramusical debido a su lugar fundacional en el desarrollo de la música popular, así como a la importancia de la espontaneidad en su interpretación. Además, la accesibilidad de sus formas es especialmente apropiada para el encuentro creativo e improvisado entre músicos expertos y principiantes ${ }^{3}$. En este sentido, el prestigioso trompetista de jazz Wynton Marsalis se ha referido a la constitución del blues como lugar de encuentro y entendimiento común: "Es como si el blues hubiera nacido para proporcionarnos una excusa para tocar juntos, para entendernos. Cuando te veas invadido por las dudas, acude al blues. Verás que siempre está ahí’" (2010: 77). Desde su identidad afroamericana, Marsalis afirma la posibilidad extraordinaria de recurrir al blues como "la música de todos" durante una actuación o colaboración musical. Sugiere que tocar blues supone una incursión en un terreno cultural común y, de manera metafórica, una vuelta a las raíces, al hogar.

A modo de introducción, es importante señalar que la gran mayoría de los músicos especializados en Austin y Madrid asumen, tanto explícita como implícitamente, que el blues es un lenguaje musical complejo que hay que conocer en profundidad. No obstante, en Madrid se aprecia un mayor énfasis en la discusión específica y la reproducción explícita del término "lenguaje musical"4. El joven guitarrista madrileño Sergio Álvarez nos proporciona un ejemplo claro del uso habitual, ya que en la publicidad de sus clases particulares invita al alumno potencial a sumergirse en el "lenguaje del blues" a través de "escalas y [formas de] acompañamiento"; a aprender "a tocar como los grandes: B.B. King, Albert King, Johnny

3. Como ejemplo de esta espontaneidad y accesibilidad podemos señalar que, mientras los músicos de baile con repertorio de jazz en los que centran Faulkner y Becker (2009: 38-42) recurren a partituras y a libros de referencia como el Real Book para llegar a acuerdos en la interpretación, en el ámbito del blues no se exige leer notación musical (muchos de los referentes históricos no sabían) y el nivel de conocimientos teóricos necesario para interpretar grupalmente es menor. Frente a la comunicación a través de símbolos de notación musical, en la interpretación del blues predomina lo que Alfred Schutz (1971: 165) llamó una "mecánica del sonido" basada en el reconocimiento de sonidos y convenciones musicales que remiten al conocimiento compartido sobre una tradición musical. No obstante, cabe señalar que, por su flexibilidad, la estructura básica del blues puede adquirir múltiples y complejas formas, habitualmente a través de su interrelación con el jazz. Peñalver Vilar (2013: 114) proporciona ejemplos sobre la evolución de las estructuras y el lenguaje empleado en la improvisación del blues, diferenciando sus usos en subgéneros de blues y jazz (classic blues; swing; be bop; jazz fusión).

4. En consonancia con las conclusiones provisionales sobre apropiación musical en Austin y Madrid (Pedro, 2015), pensamos que la distancia de la escena madrileña con respecto a la tradición original del blues ha favorecido un tipo de apropiación más especializada y conscientemente analítica. 
Winter, Otis Rush..."; y a familiarizarse con los subgéneros de "Chicago, Texas y West Coast" (post publicado en Facebook, 25/01/2016). También se refiere a la posibilidad de aproximarse al funk, el rock y el jazz desde el blues. De este modo, el lenguaje musical del blues se entiende principalmente en relación a la dimensión melódica y solista del género, así como al tipo de acompañamiento instrumental que lo caracteriza y a las estructuras armónicas propias del blues (si bien no aparecen nombradas). Asimismo, la invitación a "tocar como los grandes" se relaciona con la imitación y el diálogo con voces musicales de la tradición. Por último, las referencias a géneros afines como el funk, el rock y el jazz tienen que ver con la constitución del blues como punto de referencia fundacional de la música popular, además de con la búsqueda de mayores oportunidades de negocio.

En la primera parte del artículo expondré brevemente las principales características musicales del sistema de convenciones del blues, contextualizando sus usos y planteando ejemplos diversos. En términos semióticos, introduciré elementos de la sintaxis del blues y me aproximaré a su dimensión semántica y pragmática. En la segunda parte exploraré las formas de interacción y diálogo intramusical en mayor profundidad a través de la metáfora de la interpretación musical como conversación verbal, que nos conducirá a discusiones sobre el desarrollo de voces musicales propias.

\section{EL BLUES COMO LENGUAJE MUSICAL: SISTEMA DE CONVENCIONES E INTERACCIÓN DIALÓGICA}

Las personas que cooperan para producir un trabajo artístico se apoyan en sistemas de convenciones-acuerdos previos más o menos estandarizados que son habituales y que informan sobre lo que debe y/o puede hacerse en cada obra (Becker, 2008: 29). En este sentido, las convenciones permiten el entendimiento entre los músicos participantes y contribuyen al efecto emocional creado entre ellos, así como entre ellos y el público (Íbid.: 30). Como avanzábamos, lo que denominamos "el lenguaje del blues" se caracteriza por una serie de convenciones musicales e interaccionales, históricamente desarrolladas y reinterpretadas, que resultan fundamentales para entender su modo de expresión y comunicación. Nos centraremos en tres elementos fundamentales: las estructuras armónicas, que constituyen 
el marco o contexto de acción de una interpretación; la escala de blues como principal escala melódica, empleada en la articulación de frases y solos; y el uso de la llamada y respuesta (call and response) como forma de interacción dialógica.

\subsection{Estructuras armónicas: el contexto de acción}

En primer lugar, cabe señalar que la estructura fundamental del blues es el "blues de doce compases"; una estructura cíclica sencilla (basada en la sucesión de potencialmente infinita de chorus, "vueltas" o "ruedas" en palabras de los músicos estudiados), organizada en torno a los grados I, IV y V de la escala, que en su forma más habitual se asocia al uso de acordes de séptima de dominante y a la estructura narrativa AAB. Desde la perspectiva interaccional que proponemos, basada en el uso práctico de los músicos estudiados, su principal "virtud" es su accesibilidad, flexibilidad y capacidad para crear marcos apropiados para la interacción grupal y la improvisación ${ }^{5}$. Algunos ejemplos de canciones de blues con la estructura de doce compases incluyen "Sweet Home Chicago" (Robert Johnson, 1937), "Baby What You Want Me To Do" (Jimmy Reed, 1959) y "Rock Me Baby" (B.B. King, 1964). Esta estructura de blues y los cambios armónicos I-IV-V han sido utilizados extensamente en otros géneros como el jazz, el rock 'n' roll, el soul, el rock y el pop. Así, se componen canciones con una estructura de blues (o con ligeras variaciones de ella), pero con un tratamiento rítmico y/o melódico distinto. Es decir, que una estructura de blues puede tener un ritmo de rock ' $n$ ' roll, o servir como lienzo para una improvisación jazzística.

Además del blues de doce compases, en la investigación etnográfica realizada hemos podido comprobar la interpretación práctica de otras estructuras como el blues de ocho compases, ejemplificado en la canción "Key to the Highway" (Big Bill Broonzy, 1941); el de dieciséis compases, al estilo de "Hoochie Coochie Man" (Muddy Waters, 1954); y el "blues menor", definido en la práctica por canciones como "The Thrill is Gone"

5. Entre otras formulaciones más complejas y discutidas, la improvisación musical ha sido definida como la creación espontánea de música en el transcurso de la interpretación (Monson, 2002: 114; Nettl y Russell, 2004: 9). Cabe afirmar que la improvisación se basa en el conjunto conocimientos teóricos y prácticos aprendidos por un músico, así como en su necesaria y entrenada adaptación a determinadas estructuras y lógicas musicales, a las que debe responder espontáneamente en distintas situaciones espaciotemporales. 
(B.B. King, 1969) y "As the Years Go Passing By" (1967), entre otras. La interpretación de éste último en jam sessions y conciertos de blues en Austin y Madrid resulta mucho menos habitual que la del blues en su forma más estándar, definido por una estructura mayor y una escala ambigua como la del blues -que admite notas propias de la escala mayor y menor. También cabe señalar la existencia de estructuras particulares propias de standards influyentes como "Call It Stormy Monday (But Tuesday Is Just as Bad)" (T-Bone Walker, 1947) o "Nobody Wants You When You're Down And Out" (Bessie Smith, 1929), y de canciones de blues que se mantienen en el grado I en todo su desarrollo: por ejemplo, "Crawling King Snake" (John Lee Hooker, 1949) y “Smokestack Lightnin”” (Howlin' Wolf, 1956) .

\subsection{Escala de blues: melodía, fraseo y voz musical}

Anivel melódico, el blues se caracteriza por el uso de la escala de blues y la incorporación de blue notes -notas "tristes", "azules" o "quebradas" que se utilizan como recurso expresivo para hacer inflexiones y obtener la sonoridad que es propia del género. Creada en el sur de EE.UU por los afroamericanos descendientes de esclavos, la escala de blues se considera una extensión de la escala pentatónica menor, resultado de la hibridación entre elementos africanos y europeos (Keil, 1991: 30-34; Murray, 1976: 63). El desarrollo de las blue notes, por tanto, puede entenderse desde el punto de vista de la diáspora africana (especialmente de países de África occidental como Senegal, Gambia y Mali) (Floyd, 1995: 75), y también apunta hacia su uso en diversos géneros de la música popular, donde ha sido apropiado por multitud de cantantes y músicos que recurren a cantos melismáticos y al estiramiento de notas durante la interpretación.

En el desarrollo histórico del blues, estas inflexiones provienen del uso de la voz como instrumento de expresión y comunicación básico y principal. Paulatinamente, esos sonidos fueron imitados y desarrollados en los instrumentos, a menudo recurriendo a artefactos como cuellos de botellas (bottlenecks), navajas o cuchillos que, empleados sobre las cuerdas

6. Para Faulkner y Becker (2009: 18-19), las canciones que -debido a su popularidad-se convierten en standards "constituyen una reserva de canciones que los intérpretes pueden tocar en el caso de que las conozcan, de que quieran interpretarlas y de que las condiciones lo permitan.” Así, el repertorio no es solo un listado previo de temas que pueden interpretarse durante la actuación sino que constituye una especie de depósito potencialmente común que se acciona y cobra vida en la interpretación colectiva. 
de la guitarra, servían para acompañar a la voz, conseguir una textura musical única y realizar interacciones de llamada y respuesta. Junto a ello cabe mencionar el desarrollo de técnicas expresivas (bendings, vibrato, glissando, tremolo, pull-off, etc.) a través de las cuales se construye y se expresa la voz musical. Véase un análisis musicológico sobre el estilo de B.B. King en Richardson (1999).

Si bien la importancia atribuida a las blue notes es unánime en los estudios académicos, los especialistas la han definido de "muy distinta manera" (Tirro, 2001: 75). Entre ellas resaltamos su identificación con el uso de la tercera menor y la séptima menor en contextos mayores; el uso de la cuarta aumentada o quinta disminuida; y las reflexiones sobre sistemas de afinación y temperamento, que aluden a una afinación baja del séptimo grado y al uso de la "tercera neutra" -una nota no prevista en la música "culta", que desprovee a las escalas mayor y menor de su carácter esencial (Reig Bravo, 2011: 93-98) ${ }^{7}$. Es decir, que el lenguaje del blues (en su articulación armónica y melódica) se caracteriza por dar cabida a combinaciones entre mayor y menor, que contradicen su tradicional dicotomía.

Así, si aceptamos provisionalmente, como estrategia operativa, la convención cultural básica que asocia el sonido mayor con lo alegre y el menor con lo triste (presente, por ejemplo, en Frith, 1996 y Curtis y Bharucha, 2010), podríamos sugerir que su creación original en el plano musical representaba, en cierta medida, la propia comprensión vital de los afroamericanos, marcada - más que por su oposición- por la continuidad de alegría y tristeza como aspectos entrelazados y constitutivos del mismo flujo experiencial. Con ello, aun reconociendo la ambigüedad que necesariamente implica asociar emociones a la música, apuntamos hacia la relevancia del contexto represivo de discriminación racial en el que se desarrolló el blues (Jim Crow), al sentimiento de tristeza del que emerge, y también al desarrollo del género como una forma de diversión, evasión, catarsis y "contradeclaración” (Murray, 1976: 45, 54, 68).

7. Reig Bravo (2011) explica con claridad las diferencias entre el sistema temperado, surgido en el seno de la llamada música "culta", y la afinación atemperada o "expresiva", asociada a músicas de tradición vocal. 


\subsection{La llamada y respuesta: interacción dialógica en la interpretación musical}

La llamada y respuesta es una forma de interacción dialógica originaria de la tradición cultural africana y con un papel fundamental en el desarrollo de la música afroamericana y la música popular occidental. Como característica esencial de la tradición afroamericana, presente en las canciones de trabajo y los espirituales (principales antecedentes del blues), así como en el hip hop contemporáneo, entre otros muchos géneros, la llamada y respuesta se ha entendido como un puente de unión entre la música y otras formas de expresión cultural que, junto a la improvisación, el montaje y la dramaturgia, proporcionan claves hermenéuticas sobre el abanico completo de prácticas artísticas negras (Gilroy, 1999: 78). Además, como modelo de interacción dialógica, la llamada y respuesta "simboliza y anticipa (pero no garantiza) relaciones sociales nuevas y no-dominantes", recordando que en su práctica participativa hay un momento democrático y comunitario que permite desarrollar tradiciones artísticas a través de "intensos y, a menudo, amargos diálogos" (Gilroy, 1999: 79).

En la interpretación del blues, la interacción y comunicación dialógica entre distintas voces del grupo puede producirse de diversas maneras. En primer lugar, distinguimos un patrón de llamada y respuesta caracterizada por la llamada del líder y la respuesta al unísono de los acompañantes o, en otros términos, la llamada de una voz individual (erigida como principal) y la respuesta de una voz colectiva (resultado de la unión de las voces acompañantes). Encontramos un ejemplo claro en la canción "Manish Boy" (1955), donde Muddy Waters ejerce de cantante y líder al tiempo que la banda le acompaña con un hipnótico riff-una frase musical que se repite y que, en este caso, proporciona una contestación y un marco estable para la declaración cantada.

Un segundo tipo de interacción de llamada y respuesta se produce en la contestación y potencial conversación musical entre distintas voces instrumentales de un mismo grupo. En cierta medida este tipo de interacciones intragrupales están presentes, de manera más o menos continuada y acentuada, a lo largo de toda una interpretación musical del blues, especialmente en las cuestiones relativas a la interacción de voces vocales e instrumentales y a la dinámica musical -entendida como la 
graduación y el control colectivo de la intensidad del sonido ${ }^{8}$. Se produce cuando los miembros de un grupo asumen y desarrollan su rol con éxito, al tiempo que permanecen atentos y reaccionan a lo que cada uno de los participantes está haciendo'.

La interacción de llamada y respuesta entre voces instrumentales de un grupo se hace más explícita y se comunica con mayor claridad al público cuando se produce entre dos voces solistas como, por ejemplo, la guitarra y la armónica. Consideremos un ejemplo observado en la escena de blues de Austin. En ciertos momentos de sus actuaciones, el cantantearmonicista Birdlegg interpela verbalmente al guitarrista de su banda (que puede variar según la ocasión) diciéndole: "Come on, talk to me!" [“¡Vamos, háblame!’]. Así, realiza una llamada verbal al solista, esperando que éste, a su vez, inicie una llamada musical a través de la ejecución de una frase con la guitarra a la que el propio Birdlegg contestará de manera improvisada mediante la interpretación con la armónica. En este sentido, si bien Birdlegg (en tanto líder de la banda) inicia la llamada verbalmente, esta forma de estimular a su compañero solista le sitúa a él en el lugar del que responde (tradicionalmente el del grupo acompañante) hasta que la diferencia entre ambos roles se erosiona durante el diálogo intramusical.

Por último, encontramos un tercer tipo de interacción de llamada y respuesta entre los músicos y los públicos de música en vivo. Por una parte, el cantante-frontman o el guitarrista líder de la banda tiende a saludar al público asistente mediante una interpelación verbal más o menos entusiasta, orientada a involucrar a los aficionados. Por otra parte, a menudo se incita al público a participar en la interpretación de una canción haciendo palmas con las manos o respondiendo, repitiendo o completando ciertas frases y sonidos de la canción. Si bien su éxito es variable en la práctica, este tipo de interpelaciones verbales y/o gestuales se usan habitualmente para atrapar

8. En la tradición de la llamada "música clásica" la dinámica está regulada e indicada en las partituras según distintos niveles de intensidad (matices dinámicos): piano (suave), mezzopiano, mezzoforte y forte (fuerte). En cambio, en el blues y en el jazz la dinámica se regula o se "cocina" de manera improvisada mediante la continuidad del diálogo intramusical entre las diferentes voces del grupo. El control y el uso performativo de la dinámica como signo de calidad musical y forma de estimular el impacto emocional de la música sobre el público es importante $\mathrm{y}$, a menudo, diferencia a músicos experimentados y amateurs.

9. Por las características y las respectivas historias de cada instrumento, los músicos de una banda tienden a cumplir distintos roles convencionales en el grupo, que condicionan tanto la forma de tocar de cada uno como las expectativas de otros músicos y públicos. Nos interesa identificar tres roles básicos de la interpretación grupal en el blues: el de cantante en tanto frontman y líder (canónico), que da indicaciones al resto y distribuye los solos; el de los solistas; y el de la sección rítmica. 
la atención, elevar el ánimo y generar, aunque sea provisionalmente, un tipo de interacción más participativa y comunitaria entre los músicos y el público.

\section{DIÁlOGO INTRAMUSICAL Y METÁFORA DE LA CONVERSACIÓN}

En esta sección evaluaremos con mayor detenimiento los procesos de diálogo e interacción intramusical a partir de la "metáfora de la conversación", una expresión que tomamos de la etnomusicóloga Ingrid Monson (2009), que ha investigado el tema a partir de entrevistas con músicos de jazz estadounidenses. Monson señala que la metáfora de la interpretación musical como conversación verbal sugiere tanto una analogía entre la música y el habla, como un énfasis en la sociabilidad que implica la interpretación musical (Monson, 2009: 97). En este sentido, si consideramos la expresión comunicativa del habla como acción discursiva entre un enunciador y un enunciatario, la oración "tocar es como conversar" (que encontramos en la base de la metáfora de la conversación) afirma el papel de la música como forma de expresión de un sujeto individual o colectivo (enunciador) que tiende a comunicarse con uno o varios interlocutores (enunciatario) ${ }^{10}$.

Basándonos en la observación realizada y en el tipo de expresiones verbales empleadas por los participantes, proponemos diferenciar tres formas básicas de diálogo e interacción intramusical a través de las cuales se articula la metáfora de la conversación: la interpretación musical colectiva como conversación; la llamada y respuesta a dúo como conversación; y el potencial desarrollo de una voz individual propia dentro del marco del blues.

\subsection{Interpretación musical colectiva como conversación}

En lo que respecta al primer aspecto, la metáfora de la interpretación musical grupal como conversación verbal fue expresada, de manera certera, por el cantante-guitarrista blanco Woody Russell (residente en Austin):

10. Entendemos que, independientemente de la intencionalidad de los autores y los intérpretes, la interpretación musical pública (performance) es un acto comunicativo en sí mismo, que comunica a través del sonido, el discurso verbal y la comunicación no verbal (gestos, posturas, atuendos, actitudes, etc.). 
Los músicos que más admiro tocan como si estuvieran teniendo una conversación, como si estuvieran hablando entre ellos como nosotros ahora mismo. Así que no se trata de estar gritándonos los unos a los otros todo el tiempo. Hay veces en las que vas a decir algo más y veces en las que no vas a decir demasiado porque unas palabras bastarán. Creo que es exactamente la misma aproximación que tomo para tocar un blues. Estás teniendo una conversación y estás tratando de comunicar ciertos sentimientos a un oyente. Así que si quieres gritarles todo el rato, puedes hacerlo... si lo necesitas. Pero yo prefiero abordar la conversación de una forma más sutil y concentrarme en que cada nota cuente, como si estuviese cuidadosamente seleccionando las palabras que digo en una conversación. Creo que es más importante comunicarse que mostrar tu habilidad técnica (Pedro, 2014a).

Russell estableció una comparación estructural entre la interpretación musical y la conversación; entre la expresión de frases musicales y frases verbales, entre la "pronunciación" de una nota y de una palabra. Además, señaló que la forma de expresarse a través de la interpretación depende del contexto particular. Es decir, que los músicos -igual que los hablantesdeben adaptar su actuación social de manera pragmática, regulando tanto el número de notas-palabras que emplean como el volumen al que las dicen; cuánto susurran y cuánto gritan. En consonancia con nuestro planteamiento inicial, Russell también asumió que la conversación musical se produce tanto entre los músicos de un grupo como entre los músicos y los públicos. El cantante-guitarrista expresó su voluntad de comunicar "ciertos sentimientos" al público y enfatizó la importancia de abordar la conversación musical con sutileza, buscando que cada nota resulte significativa. Frente a la exhibición técnica (también asociada al "grito"), destacó que una disposición comunicativa "cuidadosa" contribuiría positivamente a la efectividad comunicativa de su propuesta musical o "mensaje".

En el ámbito de la escena de blues en Madrid, el pianista Jorge "Raspa" García y el cantante-guitarrista César Crespo coincidieron en que 
la interpretación musical es una forma particular de hablar y conversar, y subrayaron la importancia de practicar en situaciones de interacción grupal. Partiendo de su aprendizaje en lugares de interacción musical y encuentro social, "Raspa" explicó que:

Estas músicas [como el blues y el flamenco], por más que suene a tópico, donde se aprenden de verdad es tocando en la calle con la gente y en los bares, no en tu casa. Tú en tu casa puedes estudiar mucho y lo tienes claro, pero realmente cuando llegas a entender de qué va [...] es cuando conoces a la gente y ves lo que tienen que contarte. Y aunque no te lo cuenten, solamente con verles ya aprendes muchísimo (entrevista personal, Madrid, 05/03/2015).

Refiriéndose a la interpretación espontánea del blues y el flamenco como géneros afines, "Raspa" García atribuyó una mayor importancia y autenticidad a la "calle" y a la interacción grupal en bares que al estudio formal e individual -también necesario en un nivel previo ${ }^{11}$. Además, proyectó una concepción de la interpretación musical como proceso a través del cual se conocen ciertas características personales y emocionales de otros músicos, de quienes puedes aprender a través de la comunicación verbal y no verbal.

La construcción de una voz musical colectiva, conformada por la interrelación dialógica de voces individuales, se asienta sobre una serie roles y modos de expresión convencionales que han sido establecidos históricamente (como indicábamos antes, distinguimos entre cantantelíder, solistas y sección rítmica). El discurso de César Crespo evidenció su aceptación positiva de los roles interpretativos: "al final se trata de asumir el papel que tienes y, una vez tienes eso, puedes jugar por encima" (entrevista personal, Madrid, 05/03/2015). Lejos de concebirlo como una limitación, César se refirió al sistema de convenciones del blues como un punto de encuentro y de partida sólido, pero permeable a otras influencias y estilos musicales. Hay espacio y margen para la redefinición del rol instrumental, pero ésta se asienta -especialmente en una interpretación improvisada-

11. La oposición entre calle y escuela formal se encuentra también en el discurso de otros músicos de blues y jazz entrevistados, donde se alude a la calle como lugar físico y simbólico vinculado al aprendizaje y a la interpretación de músicas populares. 
en el sistema de convenciones común que posibilita la interpretación colectiva en primer lugar. De lo contrario, cuando un miembro del grupo rompe una norma básica sin que los demás estén preparados, puede surgir el malentendido intramusical -una equivocación en la performance grupal fruto de la falta de entendimiento común y/o el desacuerdo sobre las normas, que contribuye a que la calidad de la interpretación musical pública se resienta.

Como en todas las conversaciones, el silencio juega un papel importante en la conversación musical que mantienen los miembros de un grupo durante la interpretación. De hecho, en la cultura del blues se valora positivamente la capacidad para utilizar el silencio como un recurso expresivo de la interpretación musical, tanto colectiva como solista. El armonicista Steve Power (residente en Austin) partió de las críticas negativas a la sencillez estructural del blues para establecer una distinción entre los participantes "desinformados" y los experimentados, y destacar la importancia del silencio y los matices:

A menudo, los desinformados critican el blues por ser una música sencilla, pero ahi reside su dificultad y su poder. Es dificil tocar algo que es tan sencillo en su estructura pero tan increíblemente matizado en su ejecución. Todo lo que escucha el músico no iniciado son tres acordes y mucho volumen [...]. No se dan cuenta de que, a menudo, la verdadera declaración está en el silencio. En esa nota larga y doblada que luego resuelve, a veces antes del beat, a veces detrás y a veces justo en el punto. Tensión y liberación, y eso conlleva dinámica e intuición (Pedro, 2014b).

Frente a la ejecución mecánica de los tres acordes básicos (I-IV-V)y el uso del volumen alto como forma de expresión "precaria" (frecuentemente asociada al blues-rock), Power enfatizó la importancia del silencio y del juego interaccional con respecto al beat-entendido como unidad de tiempo básica de una pieza musical. Así, sugirió que el desarrollo de la capacidad para anticipar intuitivamente la dinámica grupal y responder de manera espontánea exige tiempo, dedicación y paciencia.

Como Power, tanto "Raspa" García como César Crespo incidieron 
en la importancia del silencio y se refirieron a la habilidad de "saber callarse" -mantener la voz de acompañamiento a un volumen adecuado y respetar los turnos de palabra para que la voz colectiva de un grupo sea, idealmente, una construcción coherente y unificada de la cual pueden emerger distintas individualidades. También remarcaron la importancia de escuchar, tanto para apropiar la tradición como para interpretar blues colectivamente, y destacaron la necesidad de mantener una actitud dialógica activa y respetuosa, tanto en la música como en la vida. Así, para que una conversación musical entre varios participantes constituya verdaderamente un intercambio comunicativo $-\mathrm{y}$ no una mera yuxtaposición de voces o un "diálogo de sordos"- es necesario respetar acciones básicas de la conversación que no siempre se siguen (ni el ámbito verbal ni en el musical), como la escucha atenta, la respuesta fática y la exposición ordenada de opiniones.

\subsection{Llamada y respuesta a dúo como conversación}

La metáfora de la conversación se encuentra también en la interacción dialógica de la llamada y respuesta entre dos miembros de un grupo. Por una parte, en un nivel próximo a la "interpretación colectiva como conversación" encontramos la interacción constante entre instrumentos como la guitarra y la batería, negociada dialógicamente durante el flujo temporal de una actuación. La labor del baterista consiste en crear una pauta rítmica común y en interactuar con el solista respondiendo a sus fraseos, adivinando sus intenciones, transmitiéndole fuerza para que suba la intensidad o, por el contrario, percibiendo su voluntad de bajar la dinámica para expresarse de un modo más sutil.

Por otra, cuando la interacción de llamada y respuesta se produce entre dos solistas asistimos más claramente a una conversación musical entre dos voces individuales, que se escuchan y responden en el primer plano de la interpretación. En estos casos se advierte una similitud estructural entre el proceso de hablar y el de tocar, puesto que los dos solistas involucrados -igual que los participantes de una conversación-se responden espontáneamente en función de lo dicho por su interlocutor y de su conocimiento sobre el tema. Además, puede producirse también un contraste en el timbre de las frases que pronuncian los participantes, tanto por el sonido particular de cada instrumento como por la combinación de 
distintos registros (más graves y más agudos).

El cantante-guitarrista británico Stevie Zee (residente en Madrid) recurrió a la metáfora de la conversación para explicar las interacciones de llamada y respuesta que él y Ñaco Goñi buscan deliberadamente durante ciertos momentos de la interpretación:

Es como una conversación. Yo te digo algo y tú a veces utilizas una parte de mi frase para tu respuesta. Es un intercambio que está ocurriendo todo el tiempo, desde el principio de la canción. A veces lo acentuamos en el solo cuando, literalmente, estamos intercambiando una frase. Es interesante porque muchas veces podemos mantener una conversación con frases diferentes [...] Esa interacción es lo que nos mola porque así aprendemos cada vez más cómo somos (Pedro, 2013b).

Por una parte, Zee señaló que la interacción intramusical es constante a lo largo de una interpretación, refiriéndose así como idea de la "interpretación colectiva como conversación" ("un intercambio que está ocurriendo todo el tiempo"). Además, su referencia a la acentuación de la interacción entre voces instrumentales durante las partes improvisadas de los solos demuestra que el planteamiento de interacciones conversacionales de llamada y respuesta a dúo supone un paso más en la relación dialógica entre los participantes de una actuación grupal. En la valoración que Zee realiza de ese momento de intercambio dialógico resulta interesante destacar que la respuesta de un instrumentista puede partir de la frase de llamada realizada por su compañero. Es decir, que como en los planteamientos dialógicos de Bajtin, la voz musical propia se construye a partir de las palabras del otro ${ }^{12}$.

Este tipo de conversación musical a dúo tiende a producirse sobre un marco rítmico y armónico creado por la batería y el bajo, instrumentos que -por sus respectivos roles en el grupo- juegan un papel fundamental en la creación y mantenimiento de un patrón rítmico dinámico, aunque esencialmente estable, sobre el cual se desarrollan nuevas capas sonoras y

12. Cabe recordar que la aproximación musical e interpersonal que caracteriza a la llamada y respuesta conversacional se produce sobre la base de un consenso estilístico previo, acordado entre las voces que lideran formalmente la formación (en este caso, Stevie Zee y Naco Goñi). 
tratamientos melódicos y solistas. "Escoltados" por la sección rítmica, los diálogos de llamada y respuesta a dúo suponen un proceso de acercamiento mutuo y búsqueda de entendimiento entre los músicos involucrados, que deben adaptar su habla instrumental al flujo temporal de la canción y a las intervenciones de su interlocutor, reaccionando espontáneamente y avanzando hacia la intensificación musical. En la práctica cotidiana, la interacción conversacional a dúo tiene formas y resultados variables, incluso cuando el diálogo intramusical se produce entre dos músicos conocidos que tocan juntos habitualmente.

Esta variación evidencia que la conversación musical, como la verbal, está condicionada por las circunstancias espaciotemporales en las que tiene lugar, así como por los estados de ánimo y el entendimiento cambiante de los participantes. Es decir, que si bien el tipo de interacción puede ser practicada con regularidad (y la propia actuación puede ser registrada fácilmente a través de las tecnologías digitales), la interpretación y la conversación musical a dúo están indisolublemente vinculadas a la situación concreta, al "aquí" y al "ahora", a la irrepetibilidad de un momento que se escapa. Por ello, desarrollar la conversación musical con éxito supone siempre un reto; una aspiración práctica a redescubrir y transformar en la interpretación musical espontánea y parcialmente improvisada.

Con el tiempo y la experiencia, el planteamiento de este tipo de diálogos e interacciones intramusicales va naturalizándose en la performance, de manera que los músicos más experimentados desarrollan ciertos automatismos instintivos en función de pautas interpretativas aprendidas dialógicamente de la tradición y la experiencia compartida, que hacen que la comunicación verbal y gestual no sea tan necesaria. Para entender esta naturalización del lenguaje musical y su manifestación práctica en la interacción conversacional, es importante valorar, además del talento y la experiencia acumulada, la capacidad instantánea para identificar e interpretar cada situación musical ${ }^{13}$. En este sentido, Birdlegg explicó que una de las grandes cualidades de un artista está en su habilidad

13. Como cualquier encuentro social, el diálogo intramusical tiene como fundamento "la definición de la situación en que se da. Un participante sólo sabe cómo actuar una vez que ha identificado la situación, el significado del encuentro, lo cual supone un acuerdo entre los participantes sobre los temas que se pueden tratar en él, los valores a los que han de sentirse obligados, las finalidades del encuentro, los actores que puedan participar y los roles de que están investidos, y el grado de implicación y de adhesión apropiados a la situación" (Peñamarín, 1980: 144). 
para interpretar música sin aparente esfuerzo o necesidad de pensar, para hacerlo con convicción y seguridad en lugar de intentarlo, para fundirse finalmente con la propia música al comunicarla y encarnarla públicamente -al ser identificado inequívocamente con ella (Pedro, 2016). Cuando los músicos alcanzan este grado de apropiación del lenguaje del blues y su interpretación se percibe como poderosamente instintiva, los aficionados recurren verbalmente a una serie de expresiones relacionadas con la autenticidad, el ímpetu y las agallas.

En otros casos, la relación entre los miembros de un dúo musical alcanza un punto en que, como sucede a menudo en una pareja, la comunicación no verbal puede resultar más informativa y efectiva que la comunicación verbal. Es el caso de Fede Aguado y Osi Martínez, dos músicos madrileños que han tocado juntos a dúo durante más de veinte años. En una entrevista personal, Osi Martínez explicó que, después de haber tocado con un gran número de músicos a lo largo de su carrera, sentía una conexión musical especial con Fede Aguado, a quién se refirió como su "hermano de blues" (Pedro, 2013a). Incluso por encima de su relación personal -donde hay espacio para el desacuerdo-, Osi subrayó que una mirada al principio del concierto basta para conocer el estado de ánimo y la actitud de su compañero hacia la performance, y que después ya no es necesario ni mirarse para tocar. Este entendimiento musical mutuo y naturalizado les permite improvisar con mayor facilidad, controlar la dinámica conjuntamente, interpelar al público y contar historias sobre las canciones y sobre ellos mismos.

\subsection{El desarrollo de voces musicales propias}

Como advertíamos, la asunción de la metáfora de la conversación en el blues está presente en los discursos de músicos y aficionados, así como en las opiniones y valoraciones sobre grupos, músicos y actuaciones particulares. En el caso del desarrollo de voces musicales propias, podemos partir de las referencias a "hacer hablar" a un instrumento musical y a "hablar" a través de un instrumento. Al asumir y referirse a la capacidad de "hablar" musicalmente, los participantes reconocen el carácter dialógico de la interpretación y la conversación potencial entre músicos o entre músicos y públicos. En este sentido, cabe recordar la canción "Make My Guitar Talk, Talk, Talk To You" (1994), una composición de W.C. Clark ("padrino" del 
blues en Austin) en la que asume - a través de la interpretación de un relato en primera persona- la capacidad de hacer hablar a la guitarra como forma de interpelar al público y seducirlo:

I'm travelling through, I hope you love me too

I'll sing, I'll sing my heart out for you

And I'll make my guitar talk, talk, talk to you

You can dance, dance from 10 till 2

You can shout and make all your dreams come true

And I'll make my guitar talk, talk, talk to you

I'll play the blues, I'll play you a boogie or two And I'll sing a song over and over to you ${ }^{14}$.

Tanto en los casos observados en la etnografía como en la canción de Clark, el músico que es capaz de hacer hablar a un instrumento alcanza un logro musical y comunicativo de gran importancia, que refuerza su conexión emocional con el público. En la canción "Make My Guitar Talk, Talk, Talk To You" ("Hacer que mi guitarra te hable, te hable y te hable"), el narrador - un músico de gira con el que Clark queda identificado a través de la performance- anuncia su llegada, espera que el público le acoja con el mismo "amor" con el que él se dirige a ellos, y promete entregarse con dedicación, cantando desde el corazón y tocando blues y boogie-woogie. En ese encuentro y proceso de entrega, donde invita a los asistentes a que bailen y "hagan sus sueños realidad", su capacidad para "hacer hablar" a la guitarra juega un papel fundamental (el más importante si atendemos a las repeticiones y al título de la canción).

W.C. Clark también se refirió a la capacidad de hablar a través de la guitarra en sus actuaciones en directo, donde interpelaba a su guitarrista Patrick Thomas pidiéndole que le hablase: "Talk to me, Patrick" ("Háblame, Patrick") (Austin, 19/03/2016), del mismo modo en que el pianista madrileño Jorge "Raspa" García, en una jam session del grupo

14. "Estoy viajando y de paso, espero que tú me quieras también / Cantaré, cantaré para ti desde el corazón / y haré que mi guitarra te hable, te hable y te hable / Puedes bailar, bailar de diez a dos / Puedes gritar y hacer tus sueños realidad / y haré que mi guitarra te hable, te hable y te hable / Tocaré el blues, tocaré un boogie o dos / Y te cantaré una canción una y otra vez." 
The Tremendous (Madrid, 05/03/2015), interpelaba a un compañero de la sección de vientos mediante la exclamación: “ ¡habla!” A diferencia del caso previo de Birdlegg (que también decía "Talk to me"), estas interpelaciones de Clark y "Raspa" no eran una invitación a la llamada y respuesta conversacional, sino una indicación motivadora a que sus respectivos compañeros desarrollasen su solo como si estuviesen hablando, expresando sus puntos de vista en una conversación colectiva. Así, el reconocimiento del "habla musical" - concebida a partir del diálogo con la tradición, la apropiación del lenguaje del blues y de las capacidades expresivas del instrumento- remite directamente a la capacidad de desarrollar una voz propia.

En un sentido negativo, el uso de expresiones como "no me dicen nada" y "no me están contando nada" para hacer valoraciones estéticas pone de relieve la ausencia de un verdadero impacto emocional y comunicativo en la interpretación musical pública. Como ha expresado Ingrid Monson (1990: 84), decir que un solista (o una banda) no está diciendo nada es una especie de "insulto" y decir que alguien hace hablar a su instrumento es una alabanza. En los mejores casos, el músico que hace "hablar" a su instrumento ha conseguido desarrollar una personalidad musical y madurez expresiva que trascienden poderosamente el automatismo técnico y la mera imitación de sus referentes musicales. Al contrario del que "no dice nada" - pues sus palabras resultan inexpresivas, vacías, malsonantes o reiterativas para el oyente que realiza tal juicio de valor-, el músico que dota a su instrumento de una cualidad "vocal", lo utiliza como un medio y una extensión de su voz personal -distintiva y, al mismo tiempo, esencialmente híbrida.

En esta concepción del "habla musical" en tanto capacidad para expresarse de una forma personal subyacen revelaciones significativas sobre el acto y la capacidad de "hablar" en la interpretación. No se trata de una cualidad dada al alcance de cualquiera, ni tampoco vale (idealmente) hacer un ejercicio de "hablar por hablar", sino que es preciso desarrollar una forma de expresión musical artísticamente "elevada", precisamente por cierta similitud estructural y expresiva con el habla humana ${ }^{15}$. En el

15. En la cultura musical afroamericana existe una larga tradición de imitación instrumental de la voz humana. Conocida como "vocality", esta cualidad interpretativa está estrechamente vinculada a la expresión de efectos guturales como gruñidos, gemidos y gritos, y puede apreciarse en las obras de saxofonistas texanos como Arnett Cobb, Clifford Scott y King Curtis. 
habla musical no basta con saber reproducir notas y frases musicales; el instrumento debe ser apropiado, más allá del aprendizaje técnico, hasta el punto de convertirse en un medio de expresión ampliada, que traduce la sensibilidad y la personalidad de un determinado músico.

Sin duda, la búsqueda de una voz musical propia, identificable con la personalidad y la sensibilidad de un músico, constituye un reto central en la cultura del blues y en las escenas de Austin y Madrid. El multi-instrumentista Paul Oscher (primer músico blanco en tocar en la legendaria banda de Muddy Waters y actual residente de Austin) enfatizó la importancia de aspirar a que tu instrumento suene como una voz, planteando una oposición entre referentes históricos y músicos actuales. Recordando a los maestros afroamericanos que conoció junto a Muddy Waters, Oscher defendió la relevancia central del "tono" -entendido como fuerza y cualidad sonora de la expresión instrumental-y su vinculación con la capacidad para proyectar una voz compacta y convincente (entrevista personal, Austin, 24/04/2016). En contraposición con este ideal "vocal” de referencia, lamentó el uso excesivo de notas que, en su opinión, caracteriza a buena parte de los músicos actuales.

Oscher señaló que su preocupación por desarrollar una voz musical propia no fue inmediata sino que fue desarrollándose, de manera gradual, con el paso de los años. Admitió la dificultad de llegar a sonar "como uno mismo" pero identificó su estilo propio con la naturaleza particular de sus actuaciones actuales, donde toca la guitarra eléctrica, la armónica y el teclado (en ocasiones de forma simultánea). Junto a ello, destacó su capacidad para hacer "ciertas cosas que otra gente no hace" y para no tocar los temas "nota a nota", sino reconociendo lo que requiere la situación, improvisando y controlando, de manera precisa, el fraseo, el ritmo, la intensidad y la pausa. Aun así, Oscher admitió que el desarrollo de una voz musical propia sigue siendo un gran reto para él, y señaló que frecuentemente ese logro artístico era el resultado de la "ignorancia", las "limitaciones" y las "necesidades" de un músico. Puso como ejemplo al cantautor de folk-rock Bob Dylan, que sin ser un gran instrumentista, ni tener una gran voz, supo crear su propio estilo a partir de sus limitaciones.

En consonancia con la explicación de Paul Oscher, el cantanteguitarrista madrileño César Crespo admitió que, aunque estaba tratando de desarrollar su propia voz musical a través de un sonido "natural" y sin efectos en el que cada nota sea capaz "decir" algo, todavía no se 
podía hablar de un "sonido César Crespo" (Limnios, 2014). Por su parte, el guitarrista Pablo Sanpa (conversación personal, Madrid, 06/03/2014) también coincidió en su deseo de desarrollar una voz propia, refiriéndose a la idea flamenca de un "duende interior que todos tenemos" y que cuesta trabajo sacar a la superficie ${ }^{16}$. Para alcanzar esa meta, partían-como muchos otros músicos- de la exploración profunda y el estudio de la tradición del blues, concretamente de la subdivisión y el aprendizaje particular de los distintos subgéneros.

La búsqueda de una voz musical propia lleva a los músicos por diversos caminos de apropiación, evolución artística y autodescubrimiento personal, y la naturaleza tremendamente inspiradora de las voces de la tradición puede transformarse en una especie yugo cautivador, que dificulta el desarrollo individual. Así, el veterano músico afroamericano Tom "Bluesman" Hunter (residente en Austin) declaró públicamente en Facebook su incapacidad para librarse de ciertos fraseos solistas que identifica con músicos de referencia como Lightnin' Hopkins (asociado al blues rural) y B.B. King y Wayne Bennett (asociados al blues urbano):

No importa cuánto lo intento, no puedo sacudirme las influencias de los músicos a los que emulé cuando empecé a tocar la guitarra. Cuando toco en acústico siempre ejecuto, inconscientemente, varios riffs de Lightnin' Hopkins. Cuando toco en eléctrico siempre hay un toque de B.B. King y Wayne Bennett. ¡Han pasado más de sesenta años! Pero no me estoy quejando. Tan solo desearía ser la mitad de bueno que mis ídolos (post publicado en Facebook, 02/03/2015).

16. El concepto de "duende" - fundamental en la valoración de la interpretación del flamenco-cumple un papel similar a los de "alma" y "feeling" en la tradición afroamericana. El poeta Federico García Lorca (1933) reflexionó sobre el duende señalando que "no es una cuestión de facultad, sino de verdadero estilo vivo; es decir, de sangre; es decir, de viejísima cultura, de creación en acto.” Relacionó el duende con los "sonidos negros" y el "misterio" -términos históricamente asociados tanto a los gitanos como a los afroamericanos-y, citando a Goethe, lo definió como un "poder misterioso que todos sienten y que ningún filósofo explica". Así, considaremos que las nociones de "duende", "alma" y "feeling", vinculadas a la expresividad de un músico o de una interpretación, trascienden la mera acumulación de recursos técnicos para incluir -junto a ellos-cuestiones relativas a la performance, la presencia física, el gesto, la textura de la voz, la situación y el tipo de público, que contribuyen decisivamente al sentido de la interpretación musical. Véase un análisis del "rol determinante de la performance" en Bowman (2008). 
Frente a la recreación consciente de ciertas escuelas y voces musicales, Hunter señaló su voluntad de trascender esas influencias para expresarse a través de su propia voz. Sin embargo, tras toda una vida intentándolo se sentía obligado a admitir que la influencia fundacional de sus referentes particulares seguía bien presente. De esta manera, la búsqueda dialógica de una voz musical propia supone una lucha por desarrollar una identidad musical propia, que esté emancipada de su mera identificación con voces de la tradición ${ }^{17}$.

\section{CONCLUSIONES}

En la primera parte del artículo nos hemos aproximado al lenguaje del blues a través de la exposición y la discusión contextualizada de tres de sus elementos fundamentales: las estructuras armónicas; la escala melódica; y la interacción de la llamada y respuesta. Hemos destacado la sencillez y el carácter cíclico de las estructuras de blues, así como su habitual hibridación con otros géneros; la naturaleza particular de la escala de blues, marcada por la ambigua combinación de rasgos mayores y menores, así como por el uso constante de inflexiones expresivas; y hemos diferenciado las principales formas de interacción de la llamada y respuesta (la interacción entre la voz individual de un líder y la voz colectiva de un grupo que responde al unísono; la interacción entre dos voces instrumentales de un grupo; y la interacción entre músicos y públicos).

Estos elementos centrales del sistema de convenciones del blues son representativos del modo de expresión de esta música, marcada en su origen por procesos de hibridación entre tradiciones africanas y europeas, que fueron reinterpretadas en la experiencia afroamericana. Los elementos africanos se han asociado a la prevalencia del ritmo y al uso de expresiones guturales, así como a la improvisación colectiva e individual y a la participación activa del público. Por otra parte, los aspectos europeos se han asociado a elementos musicales formales, habitualmente transformados por la influencia de las características estilísticas africanas. Para comprender el desarrollo original del blues y de su lenguaje musical

17. Cabe señalar que también he observado a músicos que basan buena parte de su interpretación en la imitación de ciertas voces musicales de referencia. De hecho, la capacidad para replicar fraseos de ciertos músicos es una cualidad apreciada que muestra conocimientos específicos sobre la sintaxis musical solista. No obstante, en última instancia, prevalece la voluntad ideal de trascender la imitación. 
particular podemos recordar que la hibridación constituye

un momento de cruce de tradiciones y de creación de algo nuevo, de aceptación de lo extraño en el seno de lo propio [y] de distorsión de lo propio y lo ajeno en una producción, extraña quizá a ambas, pero que permite crear una experiencia necesaria, ubicar a personas desplazadas $y$ divididas en un mundo propio (Peñamarín, 2000: 2).

De esta manera, comprendemos que los repertorios de la cultura popular negra "estuvieron parcialmente determinados por su herencia, pero también críticamente determinados por las condiciones diaspóricas en las que cuajaron esas conexiones" (Hall, 1992: 27-28). Y, con ello, que la creación del blues tuvo una importancia central en la conformación de ese momento fundamental de descolonización en el cual "los sin-voz descubrieron que, efectivamente, tenían una historia que contar, que tenían lenguajes [verbales y musicales] que no eran los lenguajes del amo" (Hall, 1995: 30) ${ }^{18}$.

Además de contemplar el desarrollo del lenguaje musical del blues desde esta perspectiva histórica, la inclusión de testimonios actuales de músicos de las escenas de Austin y Madrid busca reflejar su incorporación e identificación consciente con la tradición del género, así como el tipo de apropiación y construcción de sentido que realizan para expresarse musicalmente a través del blues. Alejados de su contexto de desarrollo original, los sujetos estudiados encuentran en el blues una forma de expresión y comunicación inspiradora, que contribuye decisivamente a su desarrollo artístico y construcción identitaria. Desde luego, las circunstancias de sus experiencias vitales distan mucho de las de los creadores originales del blues (especialmente en el caso de los músicos blancos, tanto de Austin como de Madrid) y el sistema de convenciones del blues está hoy mucho más estandarizado, pero la apropiación respetuosa y el uso práctico de este lenguaje musical les vincula en el plano expresivo y nos conduce a la reflexión sobre la interacción y el diálogo intramusical.

18. Si los espirituales articulaban las esperanzas de los esclavos negros en términos religiosos, y las canciones de trabajo en términos laborales y de deseo, el blues creó un discurso que representaba la libertad de una manera más inmediata y accesible, en relación al viaje, las relaciones afectivas y la adquisición de bienes materiales, entre otros aspectos. 
La investigación muestra que los músicos de blues de Austin y Madrid, como los músicos de jazz de otras investigaciones (Berliner, 1994; Monson, 2009; Tsioulakis, 2013), asumen la centralidad de la interacción y la metáfora de la interpretación musical como conversación verbal en sus actividades cotidianas y trayectorias de apropiación. De acuerdo con los testimonios de los protagonistas, hemos diferenciado tres formas básicas de diálogo relacionadas con la metáfora de la conversación: la interpretación musical colectiva como conversación; la llamada y respuesta a dúo como conversación; y el desarrollo de voces musicales propias, asociado a la capacidad de "hacer hablar" y/o de "hablar" a través de un instrumento musical. En la primera hemos considerado la comparación estructural entre la interpretación musical y la conversación, así como la necesidad de practicar en situaciones de interacción grupal. Por otra parte, la llamada y respuesta conversacional a dúo puede entenderse como una traducción y reinterpretación de la comunicación verbal entre dos personas, en tanto forma básica de interacción humana. Este diálogo conversacional, donde intervienen formas de comunicación verbal y no verbal, tiende a articularse sobre un marco colectivo común, que facilita la emergencia de voces solistas y que posibilita la gradual naturalización del lenguaje del blues. Por último, la discusión sobre el desarrollo de voces musicales propias muestra el interés de los participantes por alcanzar este significativo logro, donde la capacidad para sonar (musicalmente) como uno mismo aparece como el resultado de concienzudas apropiaciones musicales y constantes búsquedas estéticas e identitarias.

Este artículo ha puesto de manifiesto la productividad y riqueza de abordar la discusión comunicativa e interaccional sobre la interpretación musical a través de la metáfora de la conversación. En este sentido, la asunción de la metáfora de la conversación por parte de músicos y aficionados de las escenas de Austin y Madrid ilustra el modo en que la metáfora "impregna la vida cotidiana", el lenguaje, el pensamiento y la acción (Lakoff y Johnson, 1995: 39). Desde la perspectiva etnográfica, semiótica y en parte musicológica planteada, esta metáfora nos ayuda a destacar y comprender la dimensión comunicativa y expresiva de la interpretación grupal frente a un público, transformando también nuestra propia percepción sobre la interpretación musical. Si, de acuerdo con Lakoff y Johnson (1995: 187), la metáfora es "un medio de estructurar nuestro sistema conceptual y los tipos de actividades cotidianas que llevamos a 
cabo", la metáfora de la interpretación como conversación es una forma de abordar la apropiación del lenguaje musical desde las prácticas y las relaciones interactivas en las que se produce.

Esta reflexión sugiere una relación de interdependencia entre la voz individual y la voz colectiva. Por una parte, la voz colectiva solamente puede constituirse a partir de la combinación de distintas voces individuales, que "hablan" en función de sus roles y modos de expresión convencionales. Por otra, la voz individual solo puede desarrollarse plenamente y llegar a constituirse como una voz musical propia en el contexto dialógico de la interpretación grupal. Esos procesos de formación musical se articulan a través de la interacción grupal cotidiana, el diálogo y la recuperación de voces de referencia de la tradición del blues. En este sentido, como señaló Bajtin, "un enunciado vivo, aparecido conscientemente en un momento histórico determinado, en un medio social determinado, no puede dejar de tocar miles de hilos dialógicos vivos, tejidos alrededor del objeto de ese enunciado por la conciencia ideológico-social" (Bajtin, 1989: 94). Es decir, que las voces musicales individuales y sus formas de expresión se construyen fundamentalmente a partir de las palabras y los recursos semióticos de otros sujetos, pues "el objeto está rodeado e impregnado de ideas generales, de puntos de vista, de valoraciones y acentos ajenos" (Bajtin, 1989: 94) ${ }^{19}$.

Cuando alguien consigue "hablar" a través de la música, se hacen presentes en su discurso las voces musicales que ha recuperado y transformado, de manera que el propio sujeto enunciador pasa también a ser híbrido y su palabra dialoga y actúa como una réplica negociada a los enunciados anteriores. Curiosamente, ese hibridismo aparece no solo como una garantía de supervivencia de la voz colectiva en la voz individual (Carvalho, 2007), sino también como un límite y una posibilidad para desarrollar la creatividad, autonomía, unicidad y "originalidad" de una voz musical que quiere ser propia, que quiere sonar "como uno mismo". Hay que alimentarse de la voz colectiva (en tanto tradición y grupo interpretativo), pero también moldearla en la búsqueda de una voz propia. De este modo, la originalidad de un músico o de un grupo aparece vinculada al desarrollo de una voz distintiva y "propia" (en la medida de lo

19. Si bien Bajtin habla del objeto como aquello de lo que trata el enunciado verbal, aquí entendemos que el objeto es la propia tradición del blues, su constitución en género musical, y también cada una de las canciones interpretables. 
posible), que se sitúa en el marco de la tradición y que, al mismo tiempo, supone un novedoso diálogo con la misma, que la renueva y extiende.

\section{REFERENCIAS BIBLIOGRÁFICAS}

BAJTÍN, M. (1989). Teoría y estética de la novela. Madrid: Taurus. BECKER, H. (2008). Art Worlds. Berkeley: University of California Press. BERLINER, P. (1994). Thinking in Jazz. The Infinite Art of Improvisation. Chicago: University of Chicago Press.

BOWMAN, R. (2008). "The determining role of performance in the articulation of meaning: the case of 'Try a Little Tenderness"'. En Analyzing Popular Music, A. Moore (ed.), 103-130. Cambridge: Cambridge University Press.

CARVALHO, J.J. (2007). "Hibridación". En Diccionario de relaciones interculturales, A. Barañano, J.L. García, M. Cátedra y M.J. Devillard (eds.), 175-178. Madrid: Editorial Complutense.

CRUCES, F. (ed.) (2001). Las culturas musicales. Lecturas de etnomusicología. Madrid: Trotta.

CURTIS, M. y BHARUCHA, J. (2010). "The Minor Third Communicates Sadness in Speech, Mirroring Its Use in Music". American Psychological Association, 10.3, 335-348.

FAULKNER, R. y BECKER, H. (2009). “Do You Know...?” The Jazz Repertoire in Action. Chicago: University of Chicago Press.

FLOYD, S. (1995). The Power of Black Music. Interpreting its History From Africa to the United States. Nueva York: Oxford University Press.

FRITH, S. (1996). Performing Rites. On the Value of Popular Music. Cambridge: Harvard University Press.

GARCÍA LORCA, F. (1933). "Teoría y juego del duende". Federicogarcíalorca.net, http://federicogarcialorca.net/obras lorca/teoria_y_juego_del_duende.htm [10/11/2016].

HALL, S. (1992). "What is 'Black' in Black Popular Culture”. En Black Popular Culture, G. Dent (ed.), 21-33. Seattle: Bay Press.

(1995). "The Local and the Global". Vertigo 5, 28-30.

KEIL, C. (1991). Urban Blues. Chicago: University of Chicago Press. 
LAKOFF, G. y JOHNSON, M. (1995). Metáforas de la vida cotidiana. Madrid: Cátedra.

LIMNIOS, M. (2014). "César Crespo: Iberian Blues Party”. Blues Gr., http://blues.gr/profiles/blogs/spanish-guitarist-c-sar-crespo-talksabout-his-routes-on-the [10/11/2016].

MONSON, I. (2009). Saying Something. Jazz Improvisation and Interaction. Chicago: University of Chicago Press.

MURRAY, A. (1976). Stomping The Blues. Nueva York: Da Capo.

NETTL, B. y RUSSELL, M. (2004). En el transcurso de la interpretación. Estudios sobre el mundo de la improvisación musical. Madrid: Akal.

PEDRO, J. (2013a). "Entrevista Osi Martínez". Sineris 9, marzo, http:// www.sineris.es/entrevista_osi_martinez.html [16/04/2017]. (2013b). "Entrevista Stevie Zee". Sineris 13, octubre, http://www. sineris.es/entrevista_stevie_zee.html [16/04/2017].

(2014a). "Woody Russell: Musical Communication". Blues Vibe, 15 de enero, https://bluesvibe.com/2014/01/15/woody-russellmusical-communication/ [16/04/2017].

(2014b). "The Steve Power Sessions (1): Working the Jelly with Matthew Robinson \& The Jelly Kings". Blues Vibe, 13 de octubre, https://bluesvibe.com/2014/10/13/the-steve-power-sessions1-working-the-jelly-with-matthew-robinson-the-jelly-kings/ [16/04/2017].

(2015). "Vidas de blues: contextos y trayectorias de apropiación en Austin y Madrid". TRANS - Revista Transcultural de Música 19, http://www.sibetrans.com/trans/public/docs/04a-trans-2015. pdf [10/11/2016].

(2016). "Conversations with Birdlegg (1): The Hardest Working Man in The Blues". Blues Vibe, 16 de julio, https://bluesvibe. com/2016/07/16/conversations-with-birdlegg-1-the-hardestworking-man-in-the-blues/ [16/04/2017].

PEÑA MARÍN, C. (1980). "Una aproximación interaccional al análisis del discurso". Revista Española de Investigaciones Sociológicas 12, 141-157, http://reis.cis.es/REIS/PDF/REIS_012_07.pdf [10/11/2016]. (2000). "Fronteras interculturales en la comunicación". Revista de Occidente 234, 43-59. 
PEÑALVER VILAR, J.M. (2011). “¿Qué es el jazz? Adaptación, modificación y transformación de los elementos musicales para la improvisación". Revista Electrónica de LEEME (Lista Europea Electrónica de Música en la Educación) 27, 35-87, https://ojs. uv.es/index.php/LEEME/article/view/9824 [16/04/2017].

(2013). "Propuesta y diseño de modelos para la investigación en el jazz". Musiker 20, 97-114, http://www.euskomedia.org/PDFAnlt/ musiker/20/20097114.pdf [16/04/2017].

REIG BRAVO, J. (2011). La música tradicional valenciana. Una aproximació etnomusicològica. Valencia: Institut Valencià de la Música.

RICHARDSON, J. (1999). "B.B. King. Analysis of the Artist's Evolving Guitar Technique". En Write Me A Few of Your Lines. A Blues Reader, S.C. Tracy (ed.), 282-307. Amherst: University of Massachusetts Press.

SCHUTZ, A. (1971). "Making Music Together: a Study in Social Relationship". En Alfred Schutz: Collected Papers II, A. Brodersen (ed.), 159-178. The Hague: Martinus Nijhoff.

TIRRO, F. (2001). Historia del Jazz Clásico. Barcelona: Robinbook.

TSIOULAKIS, I. (2013). “The Quality of Mutuality: Jazz Musicians in the Athenian Popular Music Industry". En Musical Performance in the Changing City. Post-industrial Contexts in Europe and the United States, F. Holt y C. Wergin, (eds.), 200-224. Nueva York: Routledge.

TURINO, T. (2008). Music as Social Life. The Politics of Participation. Chicago: The University of Chicago Press ${ }^{20}$.

Recibido el 27 de febrero de 2017.

Aceptado el 3 de abril de 2017.

20. Este artículo se ha escrito al amparo de un contrato predoctoral FPU financiado por el Ministerio de Educación, Cultura y Deporte. Agradezco a todos los músicos entrevistados en Austin y Madrid su inestimable colaboración en la investigación. También agradezco a Marcelo Jaume sus enriquecedores comentarios sobre la disciplina musicológica. 
